\title{
Thiamin deficiency and brain disorders
}

\author{
Roger F. Butterworth \\ Neuroscience Research Unit, Hôpital Saint-Luc (CHUM), 1058 St-Denis Street, Montreal, Quebec, Canada H2X 3J4
}

\begin{abstract}
Thiamin plays a key role in the maintenance of brain function. Thiamin diphosphate is cofactor for several enzymes involved in glucose metabolism whereas thiamin triphosphate has distinct properties at the neuronal membrane. Thiamin metabolism in the brain is compartmented between neurons and neighbouring glial cells. Thiamin deficiency is commonly encountered in severe malnutrition associated with chronic alcoholism, HIV-AIDS and gastrointestinal disease where it frequently results in Wernicke's encephalopathy (the Wernicke-Korsakoff syndrome). Wernicke's encephalopathy is severely underdiagnosed according to clinical criteria in both alcoholic and HIV-AIDS patients. Magnetic resonance imaging reveals bilateral ventricular enlargement, mammillary body atrophy and cerebellar degeneration indicative of selective neuronal loss that is characteristic of Wernicke's encephalopathy. Several mechanisms have been proposed to explain this selective loss of neurons including a cerebral energy deficit resulting from reductions in activity of thiamin diphosphate-dependent enzymes, oxidative stress and $\mathrm{N}$ methyl-D-aspartate receptor-mediated excitotoxicity. Both microglia and perivascular endothelial cells are sources of NO and oxidative stress in thiamin deficiency. Decreased activities of thiamin diphosphate-dependent enzymes (in particular $\alpha$-ketoglutarate dehydrogenase) have also been reported in neurodegenerative diseases such as Alzheimer's and Parkinson's diseases independent of patient malnutrition. In these cases, decreased activities result from direct toxic actions of oxidative stress and $\beta$-amyloid produced as part of the neuronal cell death cascade in these disorders.
\end{abstract}

Thiamin deficiency: Wernicke-Korsakoff syndrome: Alcoholism: AIDS: Neuronal cell death

\section{Introduction}

Thiamin (vitamin $\mathrm{B}_{1}$ ) is a water-soluble vitamin found in appreciable quantities in wholegrain cereals, yeast and some legumes. The thiamin content of these foods is sensitive to $\mathrm{pH}$ and to high temperatures. Thiamin deficiency remains an important healthcare issue in several world populations and the causes of thiamin deficiency in these populations include inadequate diets, the prolonged cooking of foods and the ingestion of certain foods containing significant quantities of thiaminases or anti-thiamin compounds. Populations at particularly high risk for the development of thiamin deficiency include pregnant and lactating women and their offspring. Currently, a minimum of $1 \mathrm{mg}$ thiamin/d is recommended for adults with a further $0.4 \mathrm{mg} / \mathrm{d}$ during pregnancy to accommodate maternal and fetal growth as well as increased maternal energy intake.
Subsequently, in order to account for the thiamin secretion in milk and the increased energy consumption during lactation, a further increment of $0.5 \mathrm{mg} / \mathrm{d}$ is recommended during lactation.

Other populations at risk for the development of thiamin deficiency include chronic alcoholics and patients with HIV-AIDS (Butterworth et al. 1991), as well as patients with severe gastrointestinal disorders. Displaced individuals in refugee camps as well as victims of political trade embargos may manifest a high incidence of thiamin deficiency (Butterworth, 2001).

\section{Thiamin-deficiency disorders}

The most common thiamin-deficiency disorders are beriberi and Wernicke's encephalopathy (WE; the WernickeKorsakoff syndrome). Beriberi occurs as one of two types. 
Dry beriberi is characterized by peripheral neuropathy consisting of symmetrical impairment of sensory and motor nerve conduction velocities affecting the distal segments of the limbs more seriously than the proximal ones. Wet beriberi, on the other hand, is characterized by oedema, tachycardia, cardiomegaly and congestive heart failure in addition to peripheral neuropathy. Infantile beriberi occurs in the early months of life and, as in adults, may have both cardiac and neurological manifestations. WE occurs in association with chronic alcoholism and HIV-AIDS, as well as with severe gastrointestinal disease. It is occasionally encountered in patients with persistent vomiting. In the case of alcoholics, thiamin deficiency results from a combination of factors including an inadequate dietary intake of the vitamin due to poor nutrition, poor gastrointestinal absorption of thiamin as a consequence of gastric disease and a loss of liver thiamin stores associated with alcoholic liver disease. In addition, there is convincing evidence to demonstrate that ethanol per se directly inhibits the transport of thiamin in the gastrointestinal tract. Ethanol also inhibits the phosphorylation of thiamin to its active diphosphate ester, which is required for cellular energy metabolism and synthetic functions.
WE is seriously underdiagnosed both in alcoholic and non-alcoholic patients. It has been estimated that in alcoholic patients, the diagnosis of WE is missed in up to $80 \%$ of cases (Harper, 1979). Similarly, a review of the literature describing WE in patients with HIV-AIDS revealed that $80 \%$ of cases had again not been adequately diagnosed clinically during life (Butterworth et al. 1991). The principal reason for its consistent underdiagnosis results from the overuse of the classical textbook definition of WE, which requires that a triad of neuropsychiatric symptoms (ophthalmoplegia, ataxia, global confusional state) be present for diagnosis. In practice, it is rare that this triad of symptoms is present; rather, many patients diagnosed subsequently with WE present only with psychomotor slowing or apathy. It is important to suspect thiamin deficiency in all patients with grossly impaired nutritional status associated with chronic alcoholism, HIV-AIDS, gastrointestinal disease or persistent vomiting and to administer thiamin to these patients in a timely manner.

In the meantime, a definitive diagnosis of WE can nowadays be accurately made using magnetic resonance imaging (Charness \& DeLaPaz, 1987) as shown in Fig. 1.
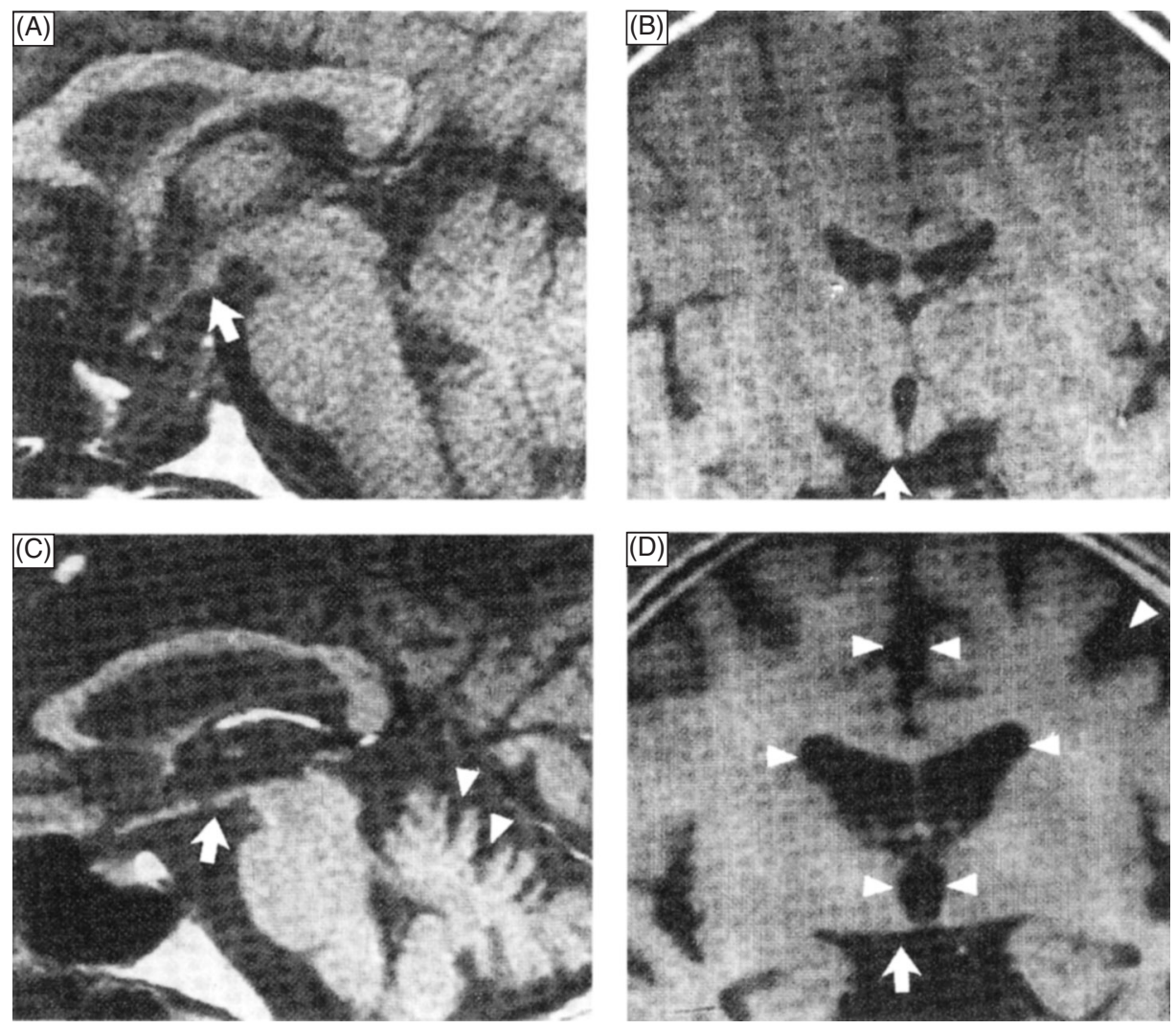

Fig. 1. Magnetic resonance imaging in a control subject ( $A$ and $B$ ) compared with a patient with chronic Wernicke's encephalopathy (WE) (C and $D)$. $T_{1}$-weighted images of sagittal $(A$ and $C$ ) and coronal (B and $D)$ sections at the level of the mammillary bodies are shown. The WE patient shows shrinkage of the mammillary bodies which is particularly apparent in the coronal section $(\leftarrow)$. Enlargement of the third and lateral ventricles is also apparent in the WE patient $(\triangle)$ confirming loss of periventricular tissue. Also apparent in the WE patient is cerebellar degeneration consisting of widening of the sulci, in the sagittal section $(\triangle)$. (Modified from Charness \& DeLaPaz, 1987.) 


\section{Thiamin and brain cell function}

Thiamin taken up into the brain is phosphorylated to thiamin diphosphate (TDP) by the enzyme thiamin pyrophosphokinase. TDP is an essential cofactor for enzymes involved in brain glucose metabolism such as transketolase, pyruvate dehydrogenase and $\alpha$-ketoglutarate dehydrogenase $(\alpha \mathrm{KGDH})$. TDP is then further phosphorylated to thiamin triphosphate (TTP) or is dephosphorylated to thiamin monophosphate (TMP). It is becoming clear that thiamin phosphorylation-dephosphorylation is a compartmented process in the brain. Evidence for this concept is derived from studies of the cellular localization of the thiamin phosphorylating and dephosphorylating enzymes as well as the phosphate esters themselves. Thiamin phosphate esters are significantly more concentrated in neurons compared with other brain cells (Laforenza et al. 1988). Moreover, TDPase activities are 20-fold higher in neurons whereas TMPase is expressed primarily by glial cells. In nerve terminals, TTP is rapidly synthesized from TDP by the action of TDP phosphoryltransferase but the TTP ester does not accumulate to high concentrations; rather it is rapidly hydrolysed to TDP by the action of TTPase, an enzyme that is also enriched in nerve terminals. Nerve stimulation results in the release of thiamin, which is mainly in the form of TMP (Cooper \& Pincus, 1979). Taken together, these findings suggest that trafficking of thiamin, and TMP occurs in the brain as shown in a simplified schematic manner in Fig. 2.
Support for this concept is derived from studies in neurological disorders such as Alzheimer's disease (AD), which is associated with significant loss of neurons and a concomitant decrease in concentrations of TDP and TDPase together with increased concentrations of TMP, consistent with reactive gliosis (Rao et al. 1993; Héroux et al. 1996). Furthermore, both ethanol and the thiamin anti-metabolite pyrithiamin cause inhibition of thiamin pyrophosphokinase and neuronal cell damage and loss.

The precise physiological role of TTP has not yet been determined but it has been proposed that the triphosphate ester activates high-conductance chloride channels (Bettendorff, 1994). TTP also has regulatory properties on proteins involved in the clustering of acetylcholine receptors (Gautam et al. 1995), suggestive of a direct role in the regulation of neurotransmission in the brain. Interestingly, rats with a high sensitivity to the central nervous system effects of ethanol express low levels of TTPase (Zimatkina et al. 2000). The eventual cloning and characterization of the genes coding for thiamin phosphorylating-dephosphorylating enzymes will no doubt stimulate further research in this interesting area and help to shed light on the precise role of TTP in brain function.

\section{Neuronal cell death due to thiamin deficiency}

Thiamin deficiency in man (WE) is characterized by a selective loss of neurons in the midbrain, thalamus and

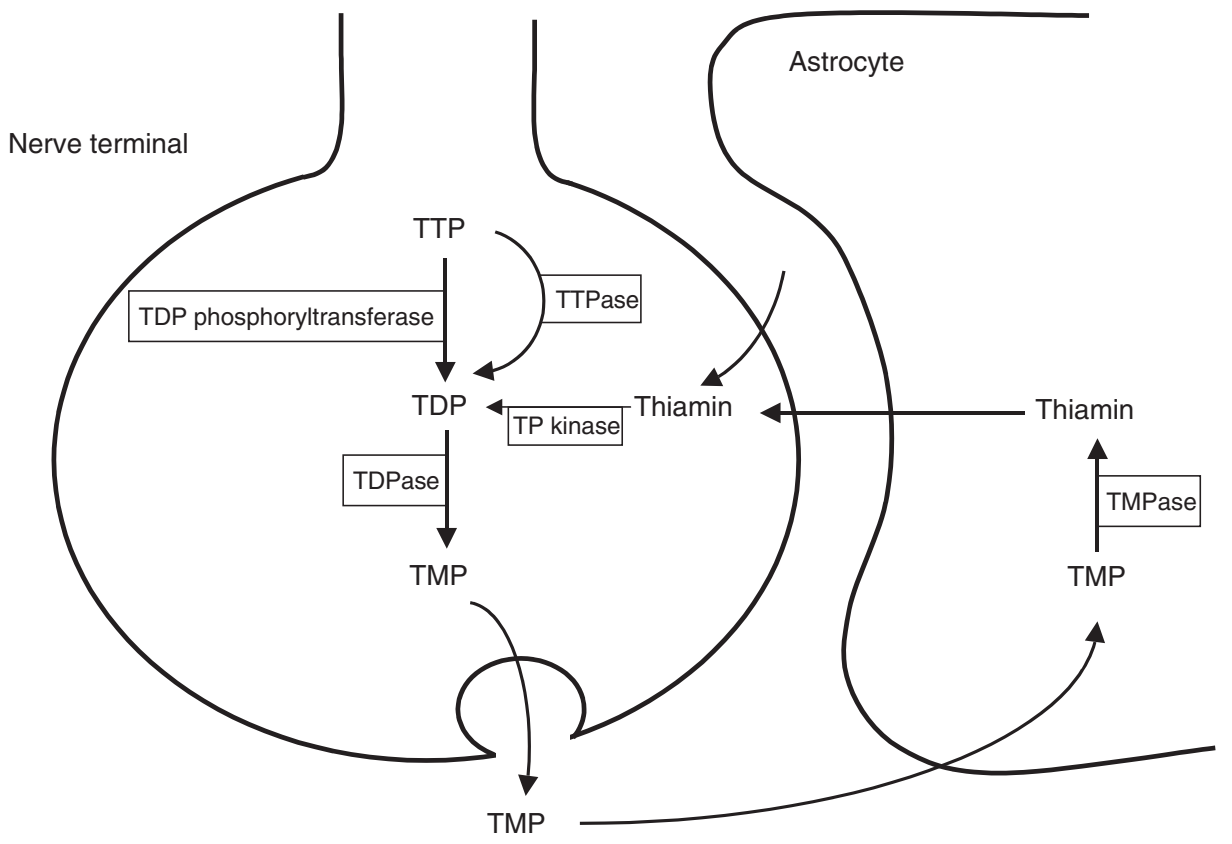

Fig. 2. Proposed trafficking of thiamin and its monophosphate ester (TMP) between nerve terminals and astrocytes in the brain. Thiamin diphosphate (TDP) is an essential cofactor for transketolase, pyruvate dehydrogenase and $\alpha$-ketoglutarate dehydrogenase, enzymes involved in brain glucose and energy metabolism. Thiamin triphosphate (TTP), on the other hand, has direct actions on neuronal membranes, particularly at the site of the chloride channel and the acetylcholine receptor. Two neuronal pools of TDP have been proposed, namely a rapid turnover pool associated with TMP and TTP synthesis and a slow turnover pool associated with cellular energy metabolism. TP kinase, thiamin pyrophosphokinase. 
cerebellum. It has been proposed that two distinct types of neuropathological damage occur in WE (Torvik, 1985), namely:

neuronal disintegration, mild endothelial swelling and sparing of the neuropil, generally confined to the thalamus and inferior olives;

destruction of the neuropil, severe endothelial swelling and neuronal sparing in mammillary bodies and periventricular brainstem nuclei.

Further insights into the nature and cause of these neuropathological features have been (and continue to be) provided by studies in experimental animal models of WE, the most popular and well characterized of which is the rat treated for $12-15 \mathrm{~d}$ with the central thiamin antagonist pyrithiamin. Such treatment reproduces both the metabolic and neuropathological features of WE. Using this experimental model, evidence of both apoptosis (Matsushima et al. 1997) and necrosis has been described. The vascular endothelium is one of the sites of early changes in the brains of pyrithiamin-treated rats (Gibson \& Zhang, 2002) followed by microglial activation (Todd \& Butterworth, 1999), breakdown of the blood-brain barrier, neuronal cell death and ensuing proliferation of reactive astrocytes. Several mechanisms have been proposed to explain the phenomenon of selective neuronal cell damage and death due to thiamin deficiency.

\section{Cellular energy failure}

Both WE in man and experimental thiamin deficiency are characterized by decreases in brain concentrations of TDP and a reduction in activities of TDP-dependent enzymes (Butterworth \& Héroux, 1989; Butterworth et al. 1993). A great deal of attention has been focused particularly on the role of decreased $\alpha \mathrm{KGDH}$ in the pathogenesis of neuronal cell death due to thiamin deficiency since it is well established that $\alpha \mathrm{KGDH}$ is a rate-limiting enzyme in the citric acid cycle, which is responsible for the maintenance of cellular energy metabolism (Fig. 3).

Prolonged reductions in activity of $\alpha \mathrm{KGDH}$ due to thiamin deficiency result in decreased glucose (pyruvate) oxidation and increased brain concentrations of alanine and lactate. Studies of oxidative metabolism in mitochondria isolated from the brains of thiamin-deficient animals reveal decreased respiration using $\alpha$-ketoglutarate as substrate but no such changes in respiration using succinate (Parker et al. 1984), findings that are consistent with decreased activities of $\alpha \mathrm{KGDH}$ (Fig. 3). Direct measurements of high-energy phosphates in the brain in experimental thiamin deficiency have been made and decreased levels of ATP described in brainstems of deficient animals (Aikawa et al. 1984). Decreased activity of $\alpha \mathrm{KGDH}$ due to thiamin deficiency also results in decreased synthesis of the important amino acid neurotransmitters glutamate and $\gamma$ amino butyric acid (Butterworth \& Héroux, 1989). Increased brain lactate con-

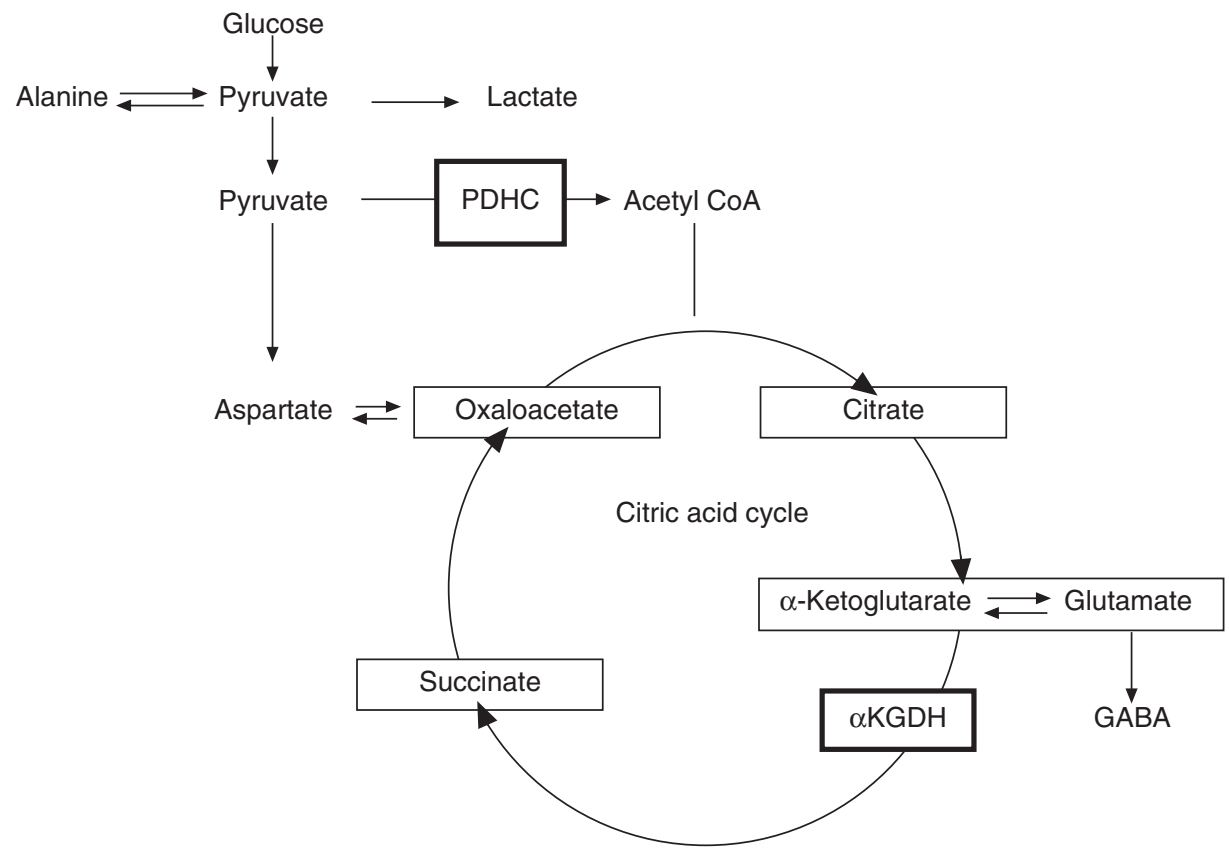

Fig. 3. Thiamin diphosphate-dependent enzymes include the pyruvate dehydrogenase complex (PDHC) and $\alpha$-ketoglutarate dehydrogenase $(\alpha \mathrm{KGDH})$ both of which are implicated in glucose oxidation and in the synthesis of the neurotransmitters glutamate and $\gamma$ amino butyric acid (GABA). Thiamin deficiency results in decreased $\alpha \mathrm{KGDH}$, and in lactate and alanine accumulation in brain; the synthesis of glutamate and GABA is concomitantly decreased. 
centrations in the brain due to thiamin deficiency were first described by Peters in the 1930s (Peters, 1936). More recently, focal accumulation of lactate leading to a reduction of tissue $\mathrm{pH}$ has been described in the brains of thiamin-deficient animals (Hakim, 1984). Moreover, cultured rat cerebellar granule cells exposed to a thiamin-deficient medium manifest decreased synthesis of TDP, decreased activities of $\alpha \mathrm{KGDH}$, increased lactate production and a concomitant reduction of cellular $\mathrm{pH}$ leading to significant cell death (Pannunzio et al. 2000). Similarly, exposure of neuroblastoma cells to the thiamin transport inhibitor amprolium results in decreased activities of $\alpha \mathrm{KGDH}$, uncoupling of mitochondria and disorganization of the cristae, all of which are restored by the addition of either thiamin or succinate (Bettendorff et al. 1995). A more prolonged exposure of these cells to amprolium led to energy compromise, depolarization and cell death. Disintegration of mitochondria has also been described in degenerating diencephalic neurons of thiamin-deficient animals (Gibson \& Zhang, 2002).

\section{Oxidative and nitrosative stress}

Tau-positive granular and fibrillary inclusions, consistent with oxidative damage, have been described in the brain in WE (Cullen \& Halliday, 1995) and increased production of reactive oxygen species has been reported in the brains of pyrithiamin-treated rats (Langlais et al. 1997). Other markers of oxidative stress in the brain in experimental thiamin deficiency include microglial activation (Todd \& Butterworth, 1999) and increased expression and activity of inducible NO synthase in these cells (Calingasan et al. 1998). Induction of inducible NO synthase results in increased nitrotyrosine immunoreactivity in regions of the brain shown ultimately to manifest neuronal cell death; nitrotyrosine is a specific nitration product of peroxynitrite, a highly potent oxidant generated by the reaction of superoxide with NO.

Vascular factors also contribute to oxidative damage to neurons in thiamin deficiency. For example, endothelial NO synthase is increased in thiamin deficiency (Fig. 4) and targeted disruption (knockout) of the endothelial NO synthase

(A)

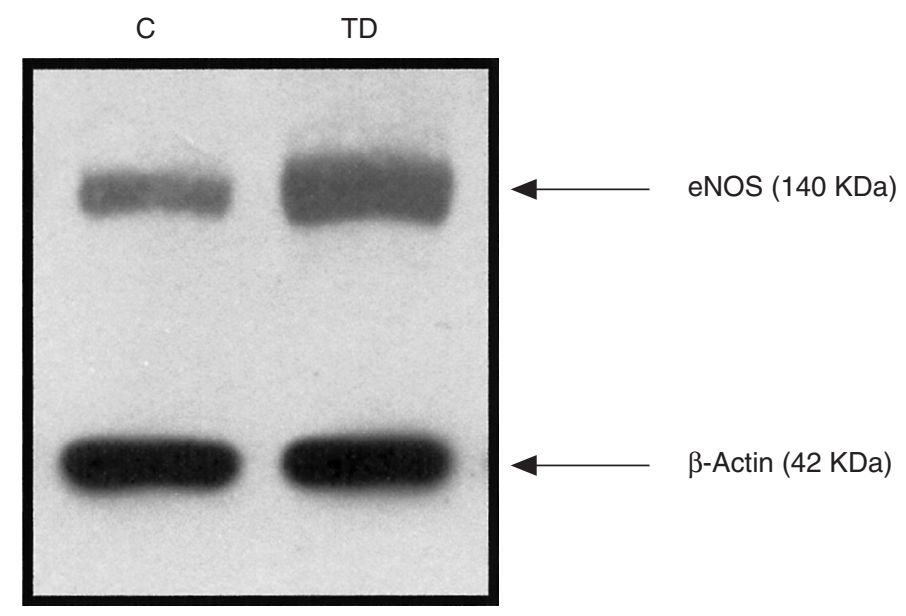

(B)
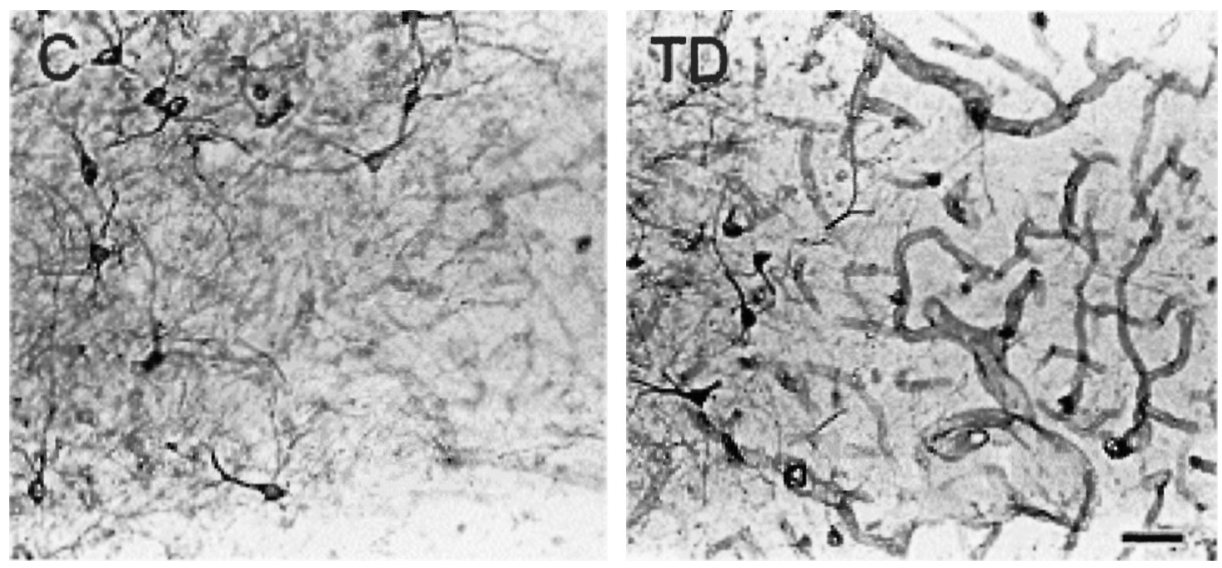

Fig. 4. Increased endothelial NO synthase (eNOS) expression in brain in experimental thiamin deficiency. (A), Increased eNOS protein in medial thalamus of a thiamin-deficient (TD) rat compared with control (C). (B), Photomicrograph showing NADPH-diaphorase staining of the inferior colliculus of a thiamin-deficient rat (TD) compared with control (C). Note the increased NADPH-immuno-straining of microvessels in TD indicative of increased eNOS activity (scale bar $=10 \mu \mathrm{m}$ ). (From Callingasan et al. 1998, with permission.) 
gene significantly attenuates the neuronal cell death due to thiamin deficiency in mice (Gibson \& Zhang, 2002). Antioxidants are neuroprotective in thiamin deficiency. For example, the cell death that accompanies the exposure of cerebellar granule cells to thiamin deficiency is significantly reduced by vitamin $\mathrm{E}$ or butylated hydroxyanisole (Pannunzio et al. 2000), both of which are powerful antioxidants. In other studies, neuronal cell loss in the thalamus, inferior colliculus and inferior olive was found to be attenuated by the free radical scavenger L-deprenyl (Todd \& Butterworth, 1998a).

Of particular importance to an understanding of cell death mechanisms implicated in the pathogenesis of neuronal cell loss due to thiamin deficiency is the report that TDP-dependent enzymes are themselves susceptible to oxidative stress. Both $\mathrm{NO}$ and peroxynitrite inactivate $\alpha \mathrm{KGDH}$ (Park et al. 1999). Thiamin deficiency also results in accumulation in the brain of amyloid precursor protein (Calingasan et al. 1995) and exposure of isolated brain mitochondria to amyloid precursor protein has been shown to result in decreased activity of $\alpha \mathrm{KGDH}$ and diminished mitochondrial respiration, which were further reduced by the addition of NO (Fig. 3) (Casley et al. 2002).

\section{$N$-methyl-D-aspartate receptor-mediated excitotoxicity}

It has been proposed that the nature of the neuropathological damage due to thiamin deficiency resembles that encountered in excitotoxic brain injury (i.e. brain injury resulting from excessive stimulation of $\mathrm{N}$-methyl-D-aspartate receptors by glutamate, a process known as excitotoxicity and shown to result in excessive accumulation of intracellular $\mathrm{Ca}$ leading to the activation of cell death mechanisms). Evidence consistent with a role for excitotoxicity in relation to neuronal cell loss due to thiamin deficiency includes the consistent finding of increased extracellular concentrations of glutamate in brain regions known to be selectively lesioned in thiamin deficiency (Hazell et al. 2001) as well as the report that pre-treatment of thiamin-deficient animals with the competitive $\mathrm{N}$ methyl-D-aspartate receptor antagonist MK801 was neuroprotective (Langlais \& Mair, 1990). However, a subsequent study found that the neuroprotective effect of MK801 was largely due to the anticonvulsant properties of the drug (Todd \& Butterworth, 1998b).

Probably the most plausible explanation for the increases of extracellular brain glutamate in thiamin deficiency relates to the finding of a selective downregulation of astrocytic glutamate transporters in brain structures vulnerable to thiamin deficiency (Hazell et al. 2001). Loss of these transporters could be related to the increase of reactive oxygen species and NO (described earlier, p. 281) since such entities are known to lead to dysfunction of these transporters (Trotti et al. 1998).

\section{Thiamin-dependent enzymes and neurodegenerative diseases}

Brain tissue from patients with $\mathrm{AD}$ contains decreased concentrations of TDP (Héroux et al. 1996) and TDPase activities are reduced by up to $60 \%$ in this material (Rao et al.
1993). Furthermore, activities of TDP-dependent enzymes have consistently been found to be decreased in AD brain (Gibson et al. 1988; Butterworth \& Besnard, 1990) with activities of $\alpha \mathrm{KGDH}$ showing particularly low levels in patients with both genetic and sporadic forms of the disease. However, in patients bearing the epsilon 4 allele of the apolipoprotein E gene (Apo E4), the correlation between $\alpha \mathrm{KGDH}$ activity and clinical dementia rating is $0 \cdot 7$ (Gibson et al. 1988). Reduced activities of the pyruvate dehydrogenase complex and transketolase have also been reported in AD (Gibson et al. 1988; Butterworth \& Besnard, 1990). Amyloid- $\beta$ peptide is an important component of senile plaques in $\mathrm{AD}$. There is increasing evidence to suggest that excess amyloid- $\beta$ peptide production is the cause of $\mathrm{AD}$ and a recent study showed that exposure of isolated brain mitochondria to amyloid- $\beta$ peptide caused a significant reduction in activities of both $\alpha \mathrm{KGDH}$ and the pyruvate dehydrogenase complex (Casley et al. 2002) suggesting that these changes contribute to the cerebral energy deficit and neuronal cell death in AD. Reduced activities of $\alpha \mathrm{KGDH}$ have also been described in other neurodegenerative diseases including Parkinson's disease (Mizuno et al. 1994) and progressive subnuclear palsy (Albers et al. 2000). As was the case in AD, it is probable that oxidative stress also plays a role in the pathogenesis of $\alpha \mathrm{KGDH}$ deficiencies in these disorders (Humphries \& Szweda, 1998).

In conclusion, WE remains a serious neuropsychiatric complication of chronic alcoholism, HIV-AIDS and severe gastrointestinal disease. The diagnosis of WE is frequently missed by current diagnostic criteria. If untreated, irreversible brain lesions in both thalamic, brainstem and cerebellar structures may occur in WE, the extent of which can be effectively assessed by magnetic resonance imaging. Studies in experimental thiamin deficiency reveal a series of probable mechanisms responsible for thiamin deficiencyrelated neuronal cell loss. Such mechanisms include cellular energy compromise, lactic acidosis, oxidative and nitrosative stress as well as N-methyl-D-aspartate receptormediated excitotoxicity. Decreased activities of thiamindependent enzymes have also been reported in the brain in neurodegenerative diseases such as AD and Parkinson's disease, findings that have been attributed to the toxic effects of oxidative stress and $B$-amyloid deposition rather than systemic thiamin deficiency in these patients.

\section{References}

Aikawa H, Watanabe IS, Furuse T, Iwasaki Y, Satoyoshi E, Sumi T \& Moroji T (1984) Low energy levels in thiamine-deficient encephalopathy. Journal of Neuropathology and Experimental Neurology 43, 276-287.

Albers DS, Augood SJ, Park LC, Browne SE, Martin DM, Adamson J, Hutton M, Standaert DG, Vonsattel JP, Gibson GE \& Beal MF (2000) Frontal lobe dysfunction in progressive supranuclear palsy: evidence for oxidative stress and mitochondrial impairment. Journal of Neurochemistry 74, 878-881.

Bettendorff L (1994) Thiamine in excitable tissues: reflections on a non-cofactor role. Metabolic Brain Disease 9, 183-210.

Bettendorff L, Sluse F, Goessens G, Wins P \& Grisar T (1995) Thiamine deficiency-induced partial necrosis and mitochondrial uncoupling in neuroblastoma cells are rapidly reversed by addition of thiamine. Journal of Neurochemistry 65, 2178-2184. 
Butterworth RF (2001) Maternal thiamine deficiency: still a problem in some world communities. American Journal of Clinical Nutrition 74, 712-713.

Butterworth RF \& Besnard AM (1990) Thiamine-dependent enzyme changes in temporal cortex of patients with Alzheimer's disease. Metabolic Brain Disease 5, 179-184.

Butterworth RF, Gaudreau C, Vincelette J, Bourgault AM, Lamothe F \& Nutini AM (1991) Thiamine deficiency and Wernicke's encephalopathy in AIDS. Metabolic Brain Disease 6, 207-212.

Butterworth RF \& Héroux M (1989) Effect of pyrithiamine treatment and subsequent thiamine rehabilitation on regional cerebral amino acids and thiamine-dependent enzymes. Journal of Neurochemistry 52, 1079-1084.

Butterworth RF, Kril JJ \& Harper CG (1993) Thiamine-dependent enzyme changes in the brains of alcoholics: relationship to the Wernicke-Korsakoff syndrome. Alcoholism, Clinical and Experimental Research 17, 1084-1088.

Calingasan NY, Gandy SE, Baker H, Sheu KFR, Kim KS, Wisniewski HM \& Gibson GE (1995) Accumulation of amyloid precursor protein-like immunoreactivity in rat brain in response to thiamine deficiency. Brain Research 677, 50-60.

Calingasan NY, Park LCH, Calo LL, Trifiletti RR, Gandy SE \& Gibson GE (1998) Induction of nitric oxide synthase and microglial responses precede selective cell death induced by chronic impairment of oxidative metabolism. American Journal of Pathology 153, 599-610.

Casley CS, Canevari L, Land JM, Clark JB \& Sharpe MA (2002) B-Amyloid inhibits integrated mitochondrial respiration and key enzyme activities. Journal of Neurochemistry 80, 91-100.

Charness ME \& DeLaPaz RL (1987) Mamillary body atrophy in Wernicke's encephalopathy: antemortem identification using magnetic resonance imaging. Annals of Neurology 22, 595-600.

Cooper JR \& Pincus JH (1979) The role of thiamine in nervous tissue. Neurochemistry Research 4, 223-239.

Cullen KM \& Halliday GM (1995) Neurofibrillary tangles in chronic alcoholics. Neuropathology and Applied Neurobiology 21, 312-318.

Gautam M, Noakes PG, Mudd J, Nichol M, Chu GC, Sanes JR \& Merlie JP (1995) Failure of postsynaptic specialisation to develop at neuromuscular junctions of rapsyn-deficient mice. Nature 377, 232-236.

Gibson GE, Sheu KF, Blass JP, Baker A, Carlson KC, Harding B \& Perrino P (1988) Reduced activities of thiamine-dependent enzymes in the brains and peripheral tissues of patients with Alzheimer's disease. Archives of Neurology 45, 836-840.

Gibson GE \& Zhang H (2002) Interactions of oxidative stress with thiamine homeostasis promote neurodegeneration. Neurochemistry International 40, 493-504.

Hakim AM (1984) The induction and the reversibility of cerebral acidosis in thiamine deficiency. Annals of Neurology 16, 673-679.

Harper CG (1979) The incidence of Wernicke's encephalopathy in Australia. A neuropathological study of 131 cases. Journal of Neurology, Neurosurgery and Psychiatry 46, 593-598.

Hazell AS, Rama Rao KV, Danbolt NC, Pow DV \& Butterworth RF (2001) Selective down-regulation of the astrocyte glutamate transporters GLT-1 and GLAST within the medial thalamus in experimental Wernicke's encephalopathy. Journal of Neurochemistry 78, 560-568.

Héroux M, Raghavendra Rao VL, Lavoie J, Richardson JS \& Butterworth RF (1996) Alterations of thiamine phosphorylation and of thiamine-dependent enzymes in Alzheimer's disease. Metabolic Brain Disease 11, 81-88.

Humphries KM \& Szweda LI (1998) Selective inactivation of alpha-ketoglutarate dehydrogenase and pyruvate dehydrogenase: reaction of lipoic acid with 4-hydroxy-2-nonenal. Biochemistry 37, 15835-15841.

Laforenza U, Patrini C \& Rindi G (1988) Distribution of thiamine, thiamine phosphates, and thiamine metabolizing enzymes in neuronal and glial cell enriched fractions of rat brain. Journal of Neurochemistry 51, 730-735.

Langlais PJ, Anderson G, Guo SX \& Bondy SC (1997) Increased cerebral free radical production during thiamine deficiency. Metabolic Brain Disease 12, 137-143.

Langlais PJ \& Mair MG (1990) Protective effects of the glutamate antagonist MK-801 on pyrithiamine-induced lesions and amino acid changes in rat brain. Journal of Neuroscience $\mathbf{1 0}$, 1664-1674.

Matsushima K, MacManus JP \& Hakim AM (1997) Apoptosis is restricted to the thalamus in thiamine-deficient rats. Neuroreport 8, 867-870.

Mizuno Y, Matuda S, Yoshino H, Mori H, Hattori N \& Ikebe S (1994) An immunohistochemical study on alpha-ketoglutarate dehydrogenase complex in Parkinson's disease. Annals of Neurology 35, 204-210.

Pannunzio P, Hazell AS, Pannunzio M, Rama Rao KV \& Butterworth RF (2000) Thiamine deficiency results in metabolic acidosis and energy failure in cerebellar granule cells: an in vitro model for the study of cell death mechanisms in Wernicke's encephalopathy. Journal of Neuroscience Research 62, 286-292.

Park LC, Zhang H, Sheu KF, Calingasan NY, Kristal BS, Lindsay JG \& Gibson GE (1999) Metabolic impairment induces oxidative stress, compromises inflammatory responses, and inactivates a key mitochondrial enzyme in microglia. Journal of Neurochemistry 72, 1948-1958.

Parker WD Jr, Haas R, Stumpf DA, Parks J, Eguren LA \& Jackson C (1984) Brain mitochondrial metabolism in experimental thiamine deficiency. Neurology 34, 1477-1481.

Peters RA (1936) The biochemical lesion in vitamin $B_{1}$ deficiency. Application of modern biochemical analysis in its diagnosis. Lancet i, 1161-1164.

Rao VL, Richardson JS \& Butterworth RF (1993) Decreased activities of thiamine diphosphatase in frontal and temporal cortex in Alzheimer's disease. Brain Research 631, 334-336.

Todd KG \& Butterworth RF (1998a) Increased neuronal cell survival after L-Deprenyl treatment in experimental thiamine deficiency. Journal of Neuroscience Research 52, 240-246.

Todd KG \& Butterworth RF (1998b) Evaluation of the role of NMDA-mediated excitotoxicity in the selective neuronal loss in experimental Wernicke encephalopathy. Experimental Neurology 149, 130-138.

Todd KG \& Butterworth RF (1999) Early microglial response in experimental thiamine deficiency: an immunohistochemical analysis. Glia 25, 190-198.

Torvik A (1985) Two types of brain lesions in Wernicke's encephalopathy. Neuropathology and Applied Neurobiology 11, 179-190.

Trotti D, Danbolt NC \& Volterra A (1998) Glutamate transporters are oxidant-vulnerable: a molecular link between oxidative and excitotoxic neurodegeneration? Trends in Pharmaceutical Science 19, 328-334.

Zimatkina TI, Chernikevich IP, Zimatkin SM \& Deitrich RA (2000) Thiamin status in liver and brain of rats genetically selected for different sensitivity to hypnotic effect of alcohol. Alcoholism, Clinical and Experimental Research 24, $1620-1624$ 


\title{
Are you a Registered Nutritionist?
}

\author{
The Nutrition Society
}

The Society's registers form part of a scheme to protect the public. The registers help to set and promote high standards of education and professional development in nutrition. The register helps employers, the media and the general public to identify appropriately qualified individuals. Members on the register are expected to adhere to the Society's Code of Ethics and the Statement of Professional Conduct. Since 2000 the status and size of the scheme has risen. Increasing numbers of employers, partner professional bodies and government departments in Britain recognise registration with the Society as a sign of quality. Members, employers and the general public can find a registered nutritionist on the Society's register at www.nutritionsociety.org/membership/register.

\section{Register of Nutritionists}

Members of the Nutrition Society can apply for Registration in Nutrition, the use of the title Registered Nutritionist and the letters $R$ Nutr after their name. Members of the Society who have been working in the field of nutrition for a minimum of three years and who have academic qualifications in nutrition, dietetics or a related subject are eligible to apply. Until 2007, other relevant qualifications may also be acceptable. Accredited Nutritionists can apply to transfer to the Society's register.

\section{Associate registration in Nutrition}

Members with an acceptable degree in nutrition or a bioscience with a major in nutrition may apply for registration as Associate Nutritionists. This confers the title Associate Nutritionist and letters A Nutr.

\section{Register of Public Health Nutritionists}

The first specialist category of the Register is the Register for Public Health Nutritionists. Members of the Society who have been working in the field of Public Health Nutrition for a minimum of three years in the last five and who have appropriate qualifications are eligible to apply. Members who fulfil the criteria for registration are able to use the title Public Health Nutritionist and the letters RPHNutr after their name.

\section{Associate registration in Public Health Nutrition}

Only members who graduated from one of the seven courses accredited by the Society are eligible for registration as Associate Public Health Nutritionists. This confers the title Associate Public Health Nutritionist and letters APHNutr.

Apply by sending

- A recent CV,

- Certified copy of qualification certificate(s) with transcripts.

- Names of THREE academic and/ or professional references

- A statement of competence

- Completed registration application form (from the website)

- The appropriate fee (see website).

Fees

The Society welcomes all applications. However, in 2004 individuals educated and resident outside the European Union will pay higher fees a reflection of the higher administration costs. Annual retention fees will not change. Registrants who lapse may be asked to pay a reinstatement fee.

For a copy of a detailed guide or information about the Registers, registration fees and about the Accreditation of Courses in Public Health Nutrition contact:

The Registrar,

The Nutrition Society,

10 Cambridge Court, 210 Shepherds Bush Rd, London W6 7NJ, ENGLAND Fax +44 (0) 207602 1756; Email: office@nutsoc.org.uk.

More details and news about developments can be found on the Society's Website: www.nutritionsociety.org.uk 